\title{
Multiphoton resonances in pulse EPR
}

\section{Conference Paper}

Author(s):

Kälin, Moritz; Gromov, Igor; Schweiger, Arthur

Publication date:

2001

Permanent link:

https://doi.org/10.3929/ethz-a-004273953

Rights / license:

In Copyright - Non-Commercial Use Permitted 


\title{
MULTIPHOTON RESONANCES IN PULSE EPR
}

\author{
Moritz Kälin, Igor Gromov, Arthur Schweiger \\ Laboratory of Physical Chemistry, ETH Zürich, CH-8093 Zürich, Switzerland
}

Reprint of the poster at the ISMAR conference 2001, Rhodes, 19 - 23 August 2001

\section{Introduction}

By using coherent rf radiation and quadrature detection we were able to improve the experiment and to distinguish between the two different absorption processes $h v_{m w}+h v_{r f}$ and $h v_{m w}-h v_{r f}$. Consequently, bichromatic pulse field-swept EPR spectra can be measured also for systems with spectra broader than the used radio frequency.

For the first time ESEEM experiments using bichromatic pulses have been carried out. In addition to the expected ESEEM spectra a peak at the radio frequency is observed. Investigations on the origin of this peak and possible schemes to remove the artefact are in progress.

Possible applications of bichromatic pulses are experiments where the exciting mw radiation interfers with the signal to be detected, e.g. in coherent Raman beat experiments or continuous wave EPR experiments. Because the excitation frequency is far off-resonant from the transition, the spin response could be separated from the exciting mw radiation. A first coherent Raman beat (CRB) experiment using a bichromatic probe pulse is presented and discussed. Examinations on cw EPR experiments applying multiphoton processes are in progress.

\section{Theory}

The discussed multiphoton processes consist of the absorption of one mw $\sigma$-photon and the absorption or emission of one or more $\mathrm{rf} \pi$-photons (Fig. 1), and may in the simplest way be described semi-classically by going into a doubly-rotating frame.

By transforming the electron spin Hamiltonian with a linearly polarised mw field (with amplitude $2 \omega_{1}$ ) perpendicular, and a linearly polarised rf field (with amplitude $2 \omega_{2}$ ) parallel to the static magnetic field to a frame rotating with frequency $\omega_{\mathrm{mw}}$, we get $\mathrm{H}=\Omega_{\mathrm{S}} \mathrm{S}_{\mathrm{z}}+\omega_{1} \mathrm{~S}_{\mathrm{x}}$

$$
+2 \omega_{2} \cos \left(\omega_{\text {rf }} t\right) S_{z}
$$

where $\Omega_{S}$ is the frequency difference between the electron

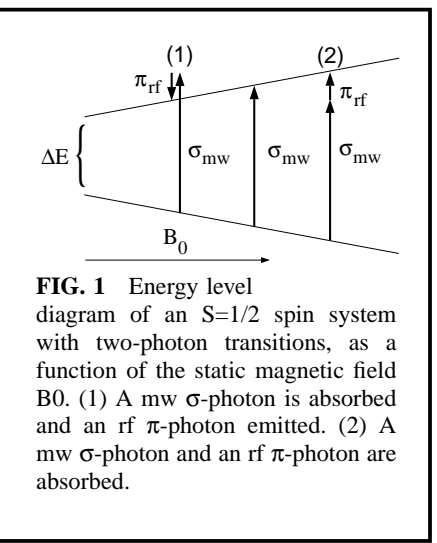

Zeeman frequency and the mw frequency. $\Omega_{\mathrm{S}}$ and $\omega_{1}$ can be combined to an effective nutation vector $\omega_{\text {eff }}$ (Fig. 2.a). The rf field is not affected by this transformation.
The frame can now be tilted by the angle $\alpha$, so that $\omega_{\text {eff }}$ is parallel to the new $\mathrm{z}$ axis, and is transformed to a doublyrotating frame, rotating with plus or minus $\omega_{\text {rf }}$ (Fig. 2.b). The part of $\mathrm{rf}$ radiation perpendicular to $\omega_{\mathrm{eff}}$ is now time independent, $\omega_{2} \sin (\alpha)$. The remaining part parallel to the $\mathrm{z}$ axis is negligible for the two-photon transition.

If the two-photon resonance condition is fulfilled, $\omega_{\text {eff }} \pm \omega_{\text {rf }}$ $=0$, the magnetisation turns around $\sin (\alpha) \omega_{2}$, in analogy to a standard mw pulse. Fig. 2.c shows the trace of the magnetisation vector in the singly-rotating frame during a bichromatic pulse. The magnetisation moves on a spiral track on the unit sphere.

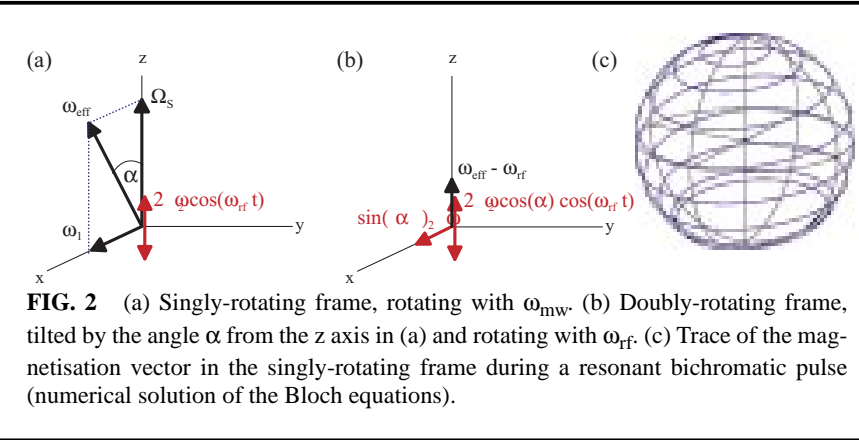

\section{Experimental}

\section{Bichromatic two-pulse echo}

The contour plot in Fig. 3 shows the Fourier transformation of a primary echo in a ruby single crystal, induced by bichromatic pulses, as a function of the static magnetic field. The peaks (1) and (2) correspond to the two transitions in Fig. 1. Since quadrature detection is applied, the two different transitions can be clearly
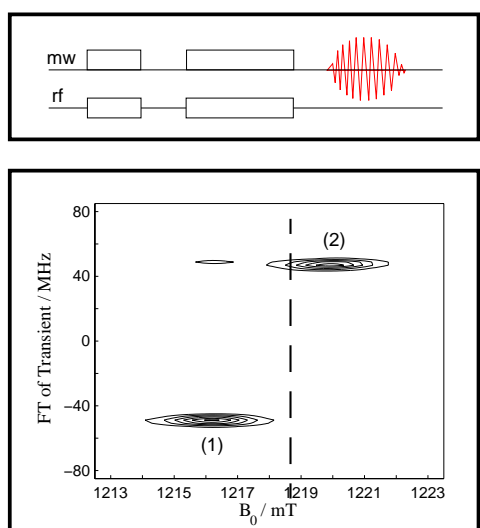

FIG. 3 Two-pulse echo experiment on a ruby single crystal, using bichromatic pulses (contour plot). $v_{\mathrm{mw}}=35.28 \mathrm{GHz}$, $v_{\mathrm{rf}}=50 \mathrm{MHz}, \mathrm{m}_{\mathrm{S}}-3 / 2 \rightarrow-1 / 2$. Abscissa: Static magnetic field. Ordinate: Fourier transformation of the transient signal of Transition $h v_{m w}+h v_{r f}$, as in Fig. 1. The position of the single-photon transition is shown by the dashed line. the echo. (1) Transition $h v_{m w}-h v_{r f}$, (2) 
separated. The zero frequency component, due to single photon transitions and dc offset, was completely supressed by applying rf phase cycling of the bichromatic pulses.

\section{Bichromatic three-pulse ESEEM}

Fig. 4.b shows a three-pulse ESEEM experiment of the ruby crystal, using bichromatic $\pi / 2$ pulses with a length of $100 \mathrm{~ns}$, at $1215 \mathrm{mT}$. For comparison the standard threepulse experiment with $20 \mathrm{~ns} \mathrm{mw}$ pulses is shown in Fig. 4.a. The spectra show the ESEEM lines of ${ }^{27} \mathrm{Al}$. The differences between the spectra of the two experiments might be caused by the smaller bandwidth of the $100 \mathrm{~ns}$ pulses and the different $\tau$ values.

Due to the rf phase dependence of the bichromatic pulses, the echo is additionally modulated with $v_{\mathrm{rf}}$, which leads to
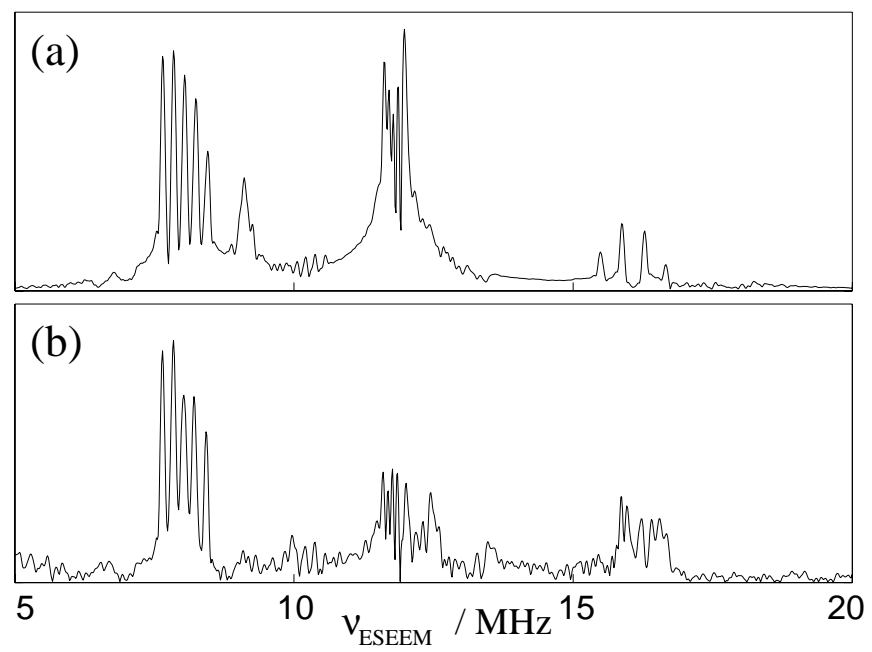

FIG. 4 (a) Standard three-pulse ESEEM spectrum of a ruby single crystal at $70 \mathrm{~K}, \mathrm{~B}_{0}=$ $1215.0 \mathrm{mT}, 20 \mathrm{~ns}$ pulses, $\tau=300 \mathrm{~ns}, v_{\mathrm{mw}}=35.28 \mathrm{GHz}$. The ${ }^{27} \mathrm{Al}$ Zeeman frequency at this field is $13.49 \mathrm{MHz}$. (b) Three-pulse ESEEM experiment with $100 \mathrm{~ns}$ bichromatic pulses, $v_{\mathrm{rf}}=40 \mathrm{MHz}, \tau=400 \mathrm{~ns}$. The applied rf is larger than any ENDOR transition.
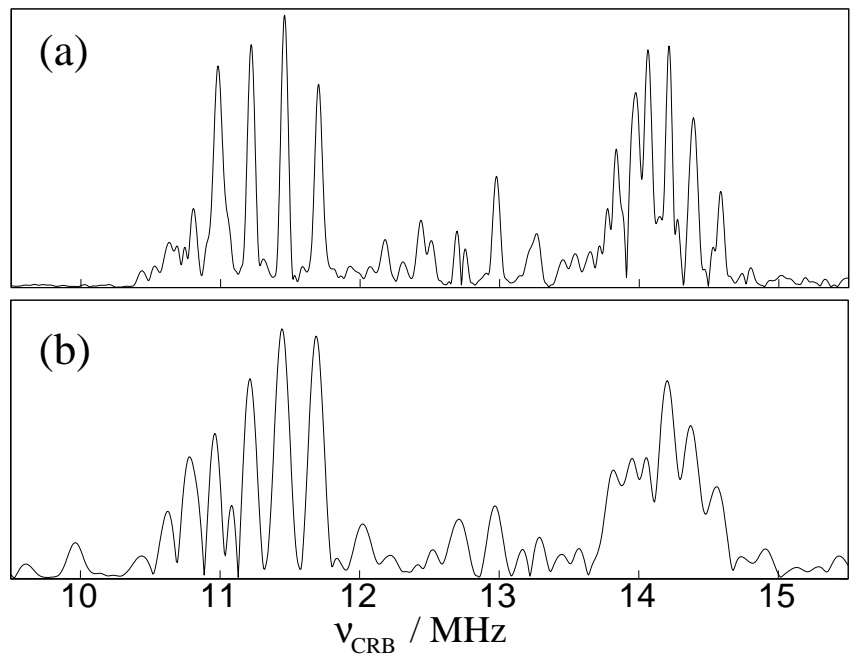

FIG. 5 Coherent Raman Beat experiments of a ruby single crystal, Fourier transfomation of the transient signals, $\mathrm{m}_{\mathrm{S}} 1 / 2->3 / 2$ transition, $\pi / 2-\tau-\pi / 2$ coherence generator sequence (12ns-140ns-12ns). (a) mw probe pulse, $\mathrm{t}_{\mathrm{P}}=33.5 \mu \mathrm{s}, \mathrm{B}_{0}=1398.79 \mathrm{mT}$. (b) Bichromatic probe pulse, $\nu_{\mathrm{rf}}=30 \mathrm{MHz}, \mathrm{t}_{\mathrm{P}}=16 \mu \mathrm{s}, \mathrm{B}_{0}=1397.41 \mathrm{mT}$. artefact peaks. The phase behaviour of the bichromatic pulses and schemes to avoid the rf artefacts are under investigation. ESEEM experiments using bichromatic pulses might be especially interesting when the applied radio frequency is additionally interacting with nuclear transitions.

\section{Coherent Raman Beat experiments using bichro- matic pulses}

Fig. 5.b shows a Coherent Raman Beat (CRB) experiment with a $\pi / 2-\tau-\pi / 2$ coherence generator sequence and a bichromatic

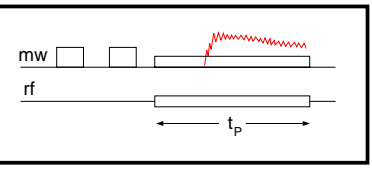

probe pulse. The advantage of using such a probe pulse would be the possibillity to filter out the reflected power of the mw probe pulse, since the detected CRB signal is shifted by $v_{\text {rf }}$ relative to the mw frequency. The short and strong $\pi / 2$ pulses excite the EPR line within a broad bandwidth, shown as blue area. The weak bichromatic probe pulse has a small bandwidth and excites mainly at

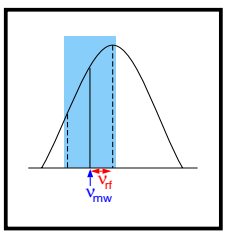
$v_{\text {mw }} \pm v_{\text {rf }}$ (dashed lines).

With a Q-band probehead it is possible to couple the mw resonator critically, which allows to minimize the reflected $\mathrm{mw}$ power and to apply the standard CRB detection scheme (it's resulting spectrum is shown for comparison in Fig. 5.a). However, at X-band and using a dielectric ring resonator this is not possible, and bichromatic CRB would be more promising.

In our experiment in Fig. 5.b we used the mw frequency as a reference for detecting the transient signal. The CRB time trace is therefore shifted by $v_{\text {rf }}$ to this mw frequency. After Fourier transformation of the CRB signal, $v_{\text {rf }}=30 \mathrm{MHz}$ was subtracted. The two CRB spectra look similar.

\section{Instrumental}

The experiments were carried out on a Q-band ( $35 \mathrm{GHz}$ ) spectrometer [2]. The original measuring system was replaced by a Bruker Elexsys E580 console, equipped with a DICE ENDOR unit, a PatternJet pulse sequence programmer and a SpecJet transient digitizer. A commercial Q-band ENDOR probehead (Bruker ER5106-QTE), turned by 90, was used.

The schematic setup for the generation of bichromatic pulses, allowing for $\mathbf{r f}$ phase cycling, is shown in Fig. 6. An rf pulse covering the whole mw pulse sequence is delivered by an arbitrary wavefunction generator (LeCroy LW420B) and

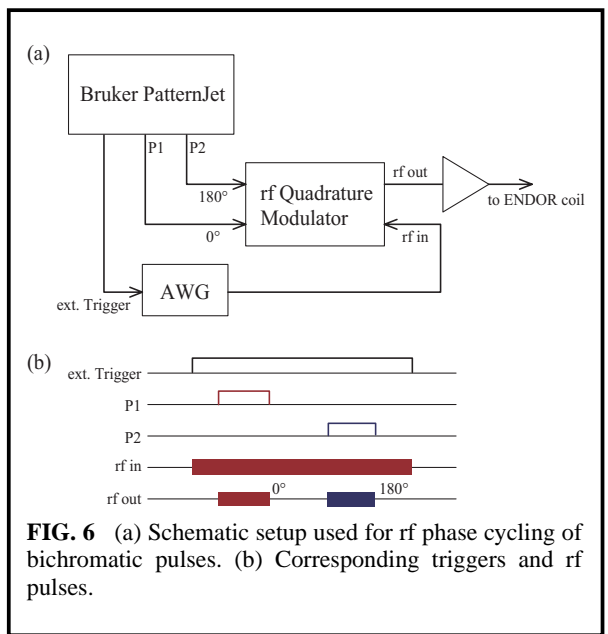


ensures an $\mathrm{rf}$ phase which is stable with respect to the pulse sequence. The rf pulses during the mw pulses (100 ns and 200 ns for $\pi / 2$ and $\pi$-pulses), are produced with a quadrature modulator, using the rf pulse triggers $\mathrm{P} 1$ and $\mathrm{P} 2$ of the PatternJet unit.

\section{Outlook}

- Investigations on the behaviour and the effects of multiphoton transitions

- Further improvement of applications of bichromatic pulses

- Development of an accurate mathematical description of multiphoton processes in EPR

\section{References}

[1] I. Gromov, A. Schweiger, J. Magn. Reson. 146, 110 (2000).

[2] I. Gromov et al., J. Magn. Reson. 149, 196 (2001). 